\title{
Uso de Substâncias em Populações de Refugiados: Propostas de Abordagem a Uma População Vulnerável
}

\section{Substance Use in Refugee Populations: Proposals for Approaching a Vulnerable Population}

\author{
Pedro MOTA $\triangle^{1}$, Ana NETO² \\ Acta Med Port 2022 Apr;35(4):239-241 - https://doi.org/10.20344/amp.16624
}

Palavras-chave: Perturbações Relacionadas ao Uso de Substâncias; Refugiados

Keywords: Refugees; Substance-Related Disorders

\section{Contextualizando a vulnerabilidade}

$\mathrm{O}$ acolhimento de um crescente número de vítimas de migração forçada na Europa tem sido acompanhado de uma reflexão sobre a necessidade e eficácia dos cuidados de saúde e intervenções disponíveis, do acesso e da utilização dos serviços de saúde, e do impacto destes na sua morbilidade a curto, médio e longo prazo., ${ }^{1,2}$ Desde 2015, Portugal recebeu, no âmbito do Programa Voluntário de Reinstalação do ACNUR (Alto-comissariado das Nações Unidas para os Refugiados), cerca de 2600 requerentes e beneficiários de proteção internacional, dos quais 1700 refugiados no âmbito de programas de recolocação europeus. Embora Portugal tenha vindo a desempenhar um papel menor neste processo, a actual organização dos serviços de Psiquiatria e de Comportamentos Aditivos e Dependências (CAD) face às necessidades específicas de saúde mental e de recursos dos refugiados são um desafio real. ${ }^{3,4}$ Acresce que a presença de enorme heterogeneidade entre estas populações implica que os serviços de saúde sejam capazes de responder adequadamente aos diversos desafios que o fenómeno migratório imprime ao processo de integração e à própria saúde mental, e com uma flexível diversidade de soluções.

Apesar da complexidade e variabilidade dos processos migratórios, as populações de refugiados aparentam ter taxas de incidência mais altas de determinadas perturbações psiquiátricas, nomeadamente de perturbação de stress pós-traumático e perturbações depressivas, ambas fatores de risco independentes para o uso de substâncias. ${ }^{1}$ Adicionalmente, estudos recentes identificam fatores de risco específicos do percurso migratório, como a exposição a eventos potencialmente traumáticos, desafios associados ao processo de integração, experiências de pobreza, discriminação e marginalização que colocam os refugiados em maior vulnerabilidade para perturbações do uso de substâncias (PUS), particularmente determinantes no período pós-migratório (Tabela 1 ). ${ }^{5} \mathrm{~A}$ prevalência das PUS, com especial destaque para a perturbação do uso de álcool, pode chegar, dependendo do contexto, a $36 \%^{2,6}$

\section{Recomendações e práticas internacionais}

Globalmente, a literatura científica relativa a intervenções para tratar PUS e reduzir os danos nestas populações é limitada, refletindo os contextos precários onde são conduzidas, tais como os campos de refugiados. ${ }^{2,6-10}$

Os verdadeiros desafios parecem centrar-se, por um lado, na transposição de rastreios e intervenções entre países com recursos e sistemas de organização de saúde distintos, e por outro, em garantir que, para além de disponíveis, os serviços sejam eficazmente adaptados de forma a torná-los sensíveis aos problemas específicos destas populações. O sucesso deste trabalho dependerá, verdadeiramente, da disponibilidade, capacidade e prontidão de estruturas e recursos humanos com competências culturais. Além disso, existe o desafio de criar intervenções que potenciem também a reconstrução e fortalecimento das comunidades afetadas, atentas aos determinantes sociais, recuperação social e cultural e integração, porque reflectem uma planificação, organização de serviços e políticas de saúde locais culturalmente sensíveis, e que requerem a mobilização de uma rede alargada de recursos humanos em saúde (administrativos, gestores, coordenadores, decisores políticos, mas também organizações não governamentais e associações de migrantes e refugiados).

No âmbito dos serviços de CAD, autores como Fedorova $(2012)^{8}$ recomendam a adaptação de princípios de intervenções culturalmente sensíveis, como a incorporação de mediadores e representantes culturais nas equipas multidisciplinares, a valorização de um papel proativo dos utentes no seu tratamento, e a exploração conjunta de sofrimento, trauma e patologias psiquiátricas eventualmente concomitantes com os CAD.

O Observatório Europeu da Droga e da Toxicodependência $(O E D T)^{2,9}$ recomenda que intervenções bem-sucedidas contemplem estratégias de (re)integração social, acesso a serviços de saúde e triagem específicos para re-

1. Serviço de Psiquiatria. Centro Hospitalar do Tâmega e Sousa. Penafiel. Portugal.

2. Unidade de Alcoologia de Lisboa. Divisão de Intervenção nos Comportamentos Aditivos e nas Dependências. Administração Regional de Saúde de Lisboa e Vale do Tejo. Lisboa. Portugal.

$\triangle$ Autor correspondente: Pedro Mota.93.pedromota@gmail.com

Recebido/Received: 28/05/2021 - Aceite/Accepted: 02/08/2022 - Publicado Online/Published Online: 14/09/2022 - Publicado/Published: 01/04/2022 Copyright @ O Ordem dos Médicos 2022 
fugiados, competência organizacional e formação adequada e que disponham de serviços de tradução e mediação. Em particular, deverá ser valorizado o recurso a materiais de informação traduzidos, privilegiando intervenções adaptadas - linguistica e contextualmente - ao utente migrante, precoces e construídas de forma colaborativa com um forte componente social e um trabalho ativo de alcance dos grupos mais vulneráveis.

No que diz respeito à estruturação da avaliação clínica, o uso de instrumentos de rastreio recomendados (AUDIT ou ASSIST) pode ser complementado pelo recurso a instrumentos que facilitam a exploração e análise conjunta de aspectos clínicos, biográficos, migratórios e culturais e da relação clínica que relacionam, preservando, o modelo biopsicosocial médico ocidental e a experiência e narrativa pessoal e cultural particulares., ${ }^{1,2,8-10}$ Destaque-se aqui a entrevista de formulação cultural do Diagnostic and Statistical Manual of Mental Disorders (DSM-5), pela comprovada utilidade diagnóstica entre clínicos e aceitabilidade entre os doentes. ${ }^{11}$

\section{QUE INTERVENÇÕES SÃO NECESSÁRIAS E POSSÍ- VEIS EM PORTUGAL?}

Avaliar as necessidades e respostas existentes. É necessário caracterizar o uso de substâncias em populações de refugiados em Portugal e harmonizar métodos de colheita de dados, em linha com as recomendações europeias. $^{2}$

Melhorar o rastreio precoce de PUS num contexto médico e cultural adequado à vulnerabilidade inicial do processo de reintegração.

Valorizar respostas longitudinais e integradas nas intervenções das equipas que acompanham os utentes no seu projecto de recolocação/reinstalação.

Partilhar, optimizar e diversificar recursos humanos. Para criar equipas técnicas verdadeiramente transdisciplinares, é necessário que médicos, enfermeiros, psicólogos e assistentes sociais sejam culturalmente competentes, mas que tradutores, mediadores culturais, representantes da comunidade e entidades associativas intervenientes tenham também formação em saúde mental e CAD. A partilha de recursos entre diversos organismos (Serviço Nacional de Saúde e Alto Comissariado para as Migrações) permitiria ultrapassar a actual escassez e centralização dos mesmos.

Promover a criação de respostas culturalmente sensíveis e adaptadas em CAD. O OEDT e outros organismos têm alertado para as dificuldades que a nível europeu condicionam a ausência destas respostas ${ }^{2,8,9}$ : carência de recursos humanos com disponibilidade e competências transculturais, dificuldade de articulação com os serviços de saúde mental e CAD, desinvestimento no rastreio e monitorização destas populações e na procura de uma ade- quada integração destas populações nos países de acolhimento. A criação destas respostas pode ser pautada por:

- Desenho de linhas orientadoras nacionais intersectoriais para Saúde Mental e CAD nesta população e criação de respostas regionais/locais que naturalmente reflitam a heterogeneidade de necessidades a nível nacional e/ou de respostas centralizadas de triagem e orientação dos casos clínicos.

- Valorização dos princípios de humanismo e pragmatismo que aproximam naturalmente os serviços de CAD dos modelos de competência cultural.

- Formação dos recursos humanos existentes quer de Saúde Mental quer de serviços de CAD, capacitando-os para o trabalho com estas populações.

- Promoção do uso de instrumentos clínicos adequados (como a formulação cultural). ${ }^{10}$

- Promover a intervenção sobre o uso de substâncias particularmente acessíveis e prevalentes, como o álcool ${ }^{2}$, e abordagem activa a grupos particularmente vulneráveis e em risco, tais como os menores não acompanhados e os refugiados a quem foi recusado o pedido de asilo, potenciais beneficiários de intervenções estruturadas de prevenção em CAD.

- Reforço da articulação com organismos governamentais e não governamentais que permita um trabalho conjunto, que complemente a intervenção e que possibilite a adesão aos serviços disponíveis.

Garantir o financiamento adequado e consistente dos diferentes níveis de intervenção mencionados.

A adaptação e implementação eficaz destas práticas - de prevenção, rastreio, referenciação, tratamento e redução de danos - melhorará a saúde e reduzirá o ónus sobre os sistemas de saúde associados às PUS. Pela sua metodologia, estas práticas beneficiarão de forma significativa todos os cidadãos, e devem ser por isso consideradas neste momento, em que simultaneamente se prevê um investimento na Saúde Mental contemplado no Plano de Recuperação e Resiliência e se discute o Plano Nacional para a Redução dos Comportamentos Aditivos e Dependências para o decénio 2021-2030.

\section{CONTRIBUTO DOS AUTORES}

PM: Conceptualização, desenvolvimento e escrita do artigo.

AN: Conceptualização, desenvolvimento e revisão do artigo

\section{CONFLITOS DE INTERESSES}

Os autores declaram não ter nenhum conflito de interesses relativamente ao presente trabalho.

\section{FONTES DE FINANCIAMENTO}

Não existiram fontes externas de financiamento ou bolsas para a realização deste trabalho. 


\section{REFERÊNCIAS}

1. Blackmore R, Boyle JA, Fazel M, Ranasinha S, Gray KM, Fitzgerald, et al. The prevalence of mental illness in refugees and asylum seekers: a systematic review and meta-analysis. PLoS Med. 2020;17:e1003337.

2. Lemmens $\mathrm{P}$, Dupont $\mathrm{H}$, Roosen I. Migrants, asylum seekers and refugees: an overview of the literature relating to drug use and access to services. Lisboa: EMCDDA; 2017

3. Neto A, Costa AG, Machado AG, Conceição D, Coutinho C, Rousseau C. Refugee resettlement I: challenges for mental healthcare services in Portugal. Acta Med Port. 2019;32:14-6.

4. Sousa T. Práticas e diálogos cruzados da psiquiatria transcultural e a população refugiada: trechos etnográficos.Lisboa: Instituto Universitário de Lisboa; 2018.

5. Cleveland J, Rousseau C, Guzder J. Cultural consultation for refugees. In: Cultural Consultation. New York: Springer; 2014. p. 245-68.

6. Horyniak D, Melo J, Farrell R, Ojeda V, Strathdee S. Epidemiology of substance use among forced migrants: a global systematic review. PLoS One. 2016;11:e0159134
7. Greene M, Kane J, Khoshnood K, Ventevogel P, Tol W. Challenges and opportunities for implementation of substance misuse interventions in conflict-affected populations. Harm Reduct J. 2018;15:58.

8. Fedorova O. Transcultural drug work: a handbook for practitioners working with drug users from different ethnic and cultural backgrounds. 2012. [consultado 2021 jan 20]. Disponível em: https://rm.coe.int/2012 3-ppg-coop-transculturalworkhandbook-web-eng/16807b6acd.

9. The European Monitoring Centre for Drugs and Drug Addiction. Drug prevention interventions targeting minority ethnic populations: issues raised by 33 case studies. 2013. [consultado 2021 jan 20]. Disponível em: https://www.emcdda.europa.eu/publications/thematic-papers/ prevention-minority-ethnic-populations_en.

10. De Kock C. Cultural competence and derivatives in substance use treatment for migrants and ethnic minorities: what's the problem represented to be? Social Theory Health. 2020;18:358-94.

11. American Psychiatric Association. Diagnostic and Statistical Manual of Mental Disorders, $5^{\text {th }}$ ed. Washington DC: APA Publishing; 2013. 